\title{
Retardation tests of inhibition following discriminative autoshaping
}

\author{
ARTHUR TOMIE and JOHN KRUSE \\ Rutgers University, New Brunswick, New Jersey 08903
}

\begin{abstract}
Four experiments explored various applications of the retardation test of inhibition following discriminative autoshaping. In all studies, discriminative autoshaping consisted of foodreinforced presentations of a green key-light CS+ and nonreinforced presentations of a vertical white line on a green background CS-. In Experiment 1, the inhibitory properties of the vertical line CS- were assessed by comparing the acquisition of keypecking to the now-reinforced vertical line $\mathrm{CS}$ in groups previously receiving discriminative autoshaping (as above), discriminative autoshaping with a red key CS-, and nondiscriminative autoshaping. The results indicated that the former CS- was retarded in being transformed into a CS+. In Experiment 2, the discriminative autoshaping procedure was followed by testing for acquisition with independent groups receiving one of four different CSs differentiated by line orientation. The results indicated that the orientation attribute of the CS- was inhibitory, in that a betweensubjects resistance-to-reinforcement gradient of inhibition was obtained. In Experiment 3, discriminative autoshaping was followed by a within-subjects resistance-to-reinforcement generalization test based on line orientation, which failed to yield an orderly gradient. The implementation of a DRO contingency in the within-subjects test (Experiment 4) was ineffective in generating an incremental gradient. Implications for inhibitory assessment methodology are discussed.
\end{abstract}

The autoshaping literature indicates that training involving negative CS-US correlation converts the key-light signal into a conditioned inhibitory stimulus. For example, Wasserman, Franklin, and Hearst (1974) have shown that when illumination of the response key is negatively correlated with the presentation of food (explicitly unpaired procedure), the key-light CS comes to elicit withdrawal from the vicinity of the signal. Furthermore, subsequent acquisition of autoshaping was retarded relative to CS-only controls. The withdrawal behavior in the presence of an explicitly unpaired CS has also been noted by Hearst and Franklin (1977), who, in addition, found that the retardation of subsequent autoshaping was directly related to the probability of food in the absence of that CS during pretraining. Analysis of the retardation effect by Wasserman and Molina (1975) has revealed that delayed initiation of keypecking and reduced postacquisition asymptotic keypecking are both induced by explicitly unpaired pretraining.

The inhibitory properties of a nonreinforced CS-

This research was supported by National Institute of Mental Health Grant MH 29425-01, National Science Foundation Grant BNS 77-20564, a Biomedical Sciences support grant administered by Rutgers University, and Rutgers Research Council grants awarded to the senior author. John Kruse is now at the Department of Psychology, University of Minnesota. Requests for reprints should be sent to Arthur Tomie, Department of Psychology, Busch Campus, Rutgers University, New Brunswick, New Jersey 08903 . following discriminative autoshaping were investigated by Wessells (1973), who exposed pigeons to differential conditioning in which a green key light $(\mathrm{CS}+)$ was followed by food and a white vertical line on a dark background (CS-) was never followed by food. In a subsequent retardation test, reinforced presentations of the former CS - and a novel white key-light CS were presented to each subject. Delayed acquisition of autoshaping to the former CS- was observed; however, there was no suppression of asymptotic levels of responding. The CS- also suppressed keypecking when combined with the CS+, and more so than for an independent group of subjects for whom the white line was novel. Therefore, summation and retardation tests indicate that the discriminative $\mathrm{CS}$ - is inhibitory.

This conclusion is supported by the results of experiments utilizing alternative indices of inhibition. For example, Wasserman et al. (1974) noted withdrawal from the vicinity of CS- during discriminative autoshaping, whereas Hearst and Franklin (1977) and Urcuioli (Note 1) obtained incremental generalization gradients around the value of $\mathrm{CS}-$ in a resistance-to-extinction test of stimulus generalization of autoshaping. In summary, the inhibitory properties of the discriminative $\mathrm{CS}-$ in autoshaping have been confirmed by the use of retardation, summation, withdrawal, and generalization tests.

The following experiments explored various applications of the retardation or resistance-to-reinforcement procedure following discriminative autoshaping. In 
all studies, discriminative autoshaping consisted of food-reinforced presentations of a green key-light $\mathrm{CS}+$ and nonreinforced presentations of a vertical white line on a green background CS-. In Experiment 1 , the inhibitory properties of the vertical line $\mathrm{CS}$ - following such discriminative autoshaping was assessed by comparing acquisition of keypecking to the new reinforced vertical line $\mathrm{CS}$ with control groups who had no prior experience with the vertical line.

In Experiment 2, dimensional inhibitory control was assessed by comparing acquisition of keypecking by independent groups of subjects to four different CSs differentiated by line orientation (betweensubjects resistance-to-reinforcement generalization gradient). In Experiments 3 and 4, dimensional inhibitory control was assessed by comparing acquisition of keypecking by a single group of pigeons to four different CSs that differed in line orientation (within-subjects resistance-to-reinforcement generalization test).

In these experiments, retarded autoshaping to the vertical line $C S$ following reliable discrimination acquisition would provide strong support for the hypothesis that discriminative autoshaping establishes the vertical line element of the CS- as a conditioned inhibitor. Alternative interpretations of such retardation based on deficits of attention to the vertical line (cf. Rescorla, 1969) would be discredited by the observation of reliable discrimination acquisition. That is, differential responding to the green $\mathrm{CS}+$ and the vertical-line-on-green $\mathrm{CS}$ - requires that the subject attend to the feature of the $\mathrm{CS}-$ that distinguishes it from the CS+. The inhibitory properties of CSfollowing discrimination acquisition in the type of differential conditioning procedure used here, therefore, may be directly evaluated by the administration of a retardation or resistance-to-reinforcement test.

\section{EXPERIMENT 1}

This experiment evaluated the retarding effects of discriminative autoshaping upon the acquisition of keypecking to the vertical line stimulus that uniquely differentiates $\mathrm{CS}-$ from $\mathrm{CS}+$. The experimental group (Group GC) was administered discrimination training in which a green key-light $\mathrm{CS}+$ was always followed by the presentation of food and the compound stimulus $(\mathrm{C})$ of a white vertical line on a green background $\mathrm{CS}-$ was never followed by the presentation of food. Control groups were administered comparable pretraining, except that nonreinforcement followed the presentation of a red key light (Group GR) or a green key light identical to CS+ (Group G.5). All groups were subsequently administered reinforced presentations of the vertical line CS on a black background, and relative rates of keypeck acquisition and asymptotic postacquisition responding were evaluated.

\section{Method}

Subjects. The subjects were 24 experimentally naive adult homing pigeons maintained at $75 \%$ of their free-feeding weights. Each subject was individually housed in a colony room with a 12-h light-dark cycle and had continuous access to grit and water.

Apparatus. Four standard pigeon conditioning chambers were used. Associated programming and recording equipment was located in an adjoining room. Each chamber measured $34 \times 33$ $\times 30 \mathrm{~cm}$ (length by width by height) and had white walls and ceiling and a stainless steel intelligence panel. A $2.9-\mathrm{cm}$-diam response key was centered on the intelligence panel $18 \mathrm{~cm}$ above a wire grid floor. Stimuli could be projected onto the response key by Industrial Electronics Engineers in-line display cells equipped with Kodak Wratten Filters 99 and $72 B$ and transilluminated by $\mathrm{CM}$ (England) 1820 miniature lamps. Ambient illumination was provided by miniature lamps located behind a $4-\mathrm{cm}$ strip of translucent Plexiglas above the intelligence panel. Masking noise was continuously provided through a 3 -in. speaker located to the right of the hopper aperture and consisted of a $200-\mathrm{Hz}$ tone measured at $95 \mathrm{~dB}$ (SPL).

Procedure. The subjects were unsystematically divided into three groups of eight pigeons each. On Day 1, each pigeon was trained to approach and eat from the food hopper. On Day 2, pigeons received the 1 st of 14 daily sessions of discriminative autoshaping. Each session lasted approximately $1 \mathrm{~h}$ and consisted of 60 trials administered according to a variable-time (VT) 45 -sec schedule. Trials consisted of the illumination of the response key for $8 \mathrm{sec}$; the presentation of CS+ was followed immediately upon offset by $4 \mathrm{sec}$ of hopper elevation, whereas the presentation of CS- was nonreinforced. Within each session, $30 \mathrm{CS}+$ and $30 \mathrm{CS}-$ trials occurred in a quasirandom order, with the restrictions that 5 of each occur in every block of 10 trials and that there be no more than three consecutive presentations of a given CS.

For all three groups, the CS+ was a green key light, but the CS- differed among groups. For the experimental group (GC), the CS - was a green key light with a superimposed vertical white line. For Group GR, the CS- was a red key light, and for Group G.5, the CS - was a green key light identical to the CS+. Following pretraining, all animals were tested for the acquisition of autoshaping to the white vertical line. On Test Day 1, each group was administered 10 trials of the discrimination, as in pretraining. This warm-up was immediately followed by 50 trials consisting of the reinforced presentation of the vertical white line key light on a dark background. Test Days 2-8 each consisted of 60 reinforced presentations of the vertical white line on the dark key. Stimulus and schedule parameters during testing were those used during pretraining.

\section{Results and Discussion}

During the pretraining phase, there were no reliable group differences in responding to the green $\mathrm{CS}+$; however, there was more responding to $\mathrm{CS}-$ by subjects in Group GC than by those in Group GR, as revealed by a mixed-design two-way analysis of variance $[F(1,14)=23.1, p<.01]$. This effect is largely attributable to high rates of responding to CS- by Group GC during the first eight sessions. There was virtually no responding to CS - by subjects in either group during the last five sessions of pretraining.

The mean acquisition functions obtained during the test phase are shown in Figure 1, which shows 


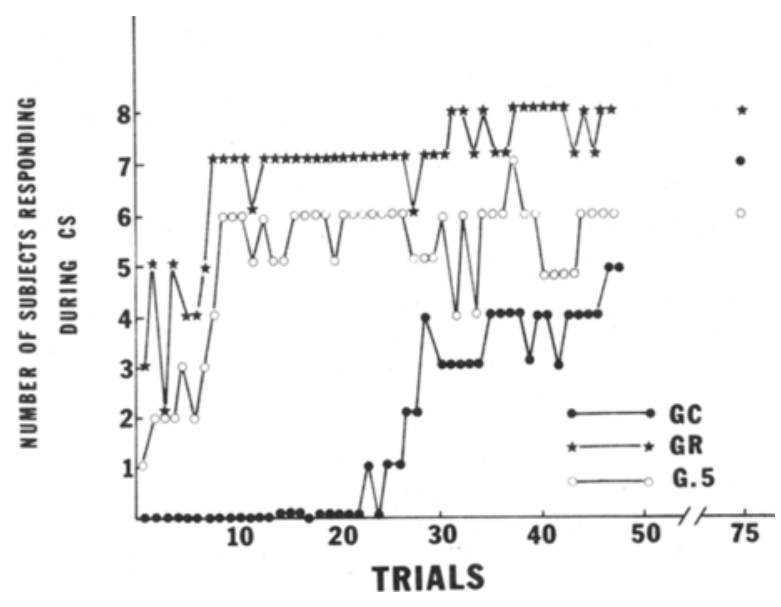

Figure 1. Number of subjects responding during $C S$ as a function of trials for Groups GC, GR, and G.5.

the number of subjects per group that keypecked at least once during the CS as a function of the first 48 test trials. As the figure reveals, subjects in Group GC were retarded in initiating keypecking, in that most of the subjects in Groups GR and G.5 were responding by Test Trial 10, whereas none of the pigeons in Group GC responded until Test Trial 22. This conclusion was supported by a two-way mixeddesign analysis of variance, which revealed a reliable main effect of groups $[\mathrm{F}(2,21)=9.98, \mathrm{p}<.01]$ and a reliable Groups by Trials interaction $[F(94,987)=1.84$, $\mathrm{p}<.01]$. A separate mixed-design analysis of variance comparing the acquisition functions of Groups $\mathrm{GC}$ and GR revealed a reliable main effect of groups $[F(1,14)=33.03, p<.01]$ and a reliable Groups by Trials interaction $[F(47,658)=1.99, p<.01]$, indicating that Group GC was retarded relative to Group GR. A similar analysis comparing the acquisition functions of Groups GC and G.5 also revealed a reliable main effect of groups $[F(1,14)=19.22$, $\mathrm{p}<.01]$ and a reliable Groups by Trials interaction $[F(47,658)=1.70, p<.01]$, indicating that Group GC was retarded relative to Group G.5. These acquisition differences were maintained only through the first 75 trials, and no asymptotic group differences were discernible (see Figure 1).

The median number of trials required by each group to attain a criterion of responding on five consecutive presentations of the white line CS provides an additional measure of the speed of acquisition. These medians were $32.5,12.5$, and 9.5 for Groups GC, G.5, and GR, respectively. A KruskalWallis analysis of variance by ranks revealed a reliable effect of groups $[\mathrm{H}(2)=8.64, \mathrm{p}<.025]$. MannWhitney $U$ tests indicated that Groups GC and GR differed reliably $(\mathrm{U}=60, \mathrm{p}<.01)$, but Groups $\mathrm{GC}$ vs. G. 5 and GR vs. G.5 did not $(U=49, .05<p<.10$, and $\mathrm{U}=41, \mathrm{p}>.10$, respectively).
The results support the following conclusions. The two groups administered discriminative autoshaping during pretraining responded differentially to the two CSs; however, more responses to CS - were observed in Group GC than in Group GR. These acquisition differences may be taken to reflect the greater similarity of the discriminative CSs in the GC as opposed to the GR discrimination. Alternatively, the differences in acquisition may be taken to reflect a featurenegative effect, since the distinctive stimulus element upon which the discrimination is based is correlated with nonreinforcement (i.e., present on $\mathrm{CS}-$ ) for Group GC. Hearst and Jenkins (1974) and Wasserman and Anderson (1974) have reported retarded discrimination formation in feature-negative situations.

The pattern of results obtained in the retardation test indicates that the acquisition of autoshaping to the vertical line CS for Group GC was reliably retarded relative to control groups that also received discriminative autoshaping during pretraining (Group GR) or nonreinforced presentations of the green CS (G.5). Retarded autoshaping to the negative stimulus element of a compound CS - suggests that the element is a conditioned inhibitor and is consistent with the notion that CS - is inhibitory following discriminative autoshaping (Hearst \& Franklin, 1977; Wessells, 1973; Urcuioli, Note 1).

These effects of pretraining were observed only upon the rate of acquisition of autoshaping. There was no evidence of group differences in asymptotic probability or frequency of keypecking. This outcome is similar to that reported by Wessells (1973) and suggests that suppression of asymptotic automaintenance following explicitly unpaired pretraining (Wasserman \& Molina, 1975) is not a general performance characteristic of subjects previously exposed to negatively correlated presentations of the CS and US.

\section{EXPERIMENT 2}

The results of Experiment 1 indicate that inhibitory control by the vertical line component of the $\mathrm{CS}-$ is established by discriminative autoshaping. Identification of precisely which attributes of that stimulus (e.g., luminosity, width, orientation) are inhibitory may be determined by evaluating dimensional control by the attribute. Inhibitory control by, for example, the orientation of the line would be revealed by an incremental generalization gradient to lines of different orientations, with a trough at the value of CS-. Retardation of acquisition, therefore, which systematically varies inversely with rotation away from vertical, would indicate dimensional inhibitory stimulus control by line orientation.

In addition to allowing for more precise specifica- 
tion of the inhibitory attributes of the CS-, a generalization test offers several other advantages over the inhibition assessment methodology employed in Experiment 1. In that experiment, the pretraining of the different control groups necessarily differed from that of the experimental group in a number of ways; therefore, test differences were subject to multiple interpretations. For example, a number of investigators (cf. Honig, 1969; Jenkins \& Harrison, 1960; Wagner, 1969) suggest that discrimination training neutralizes incidental stimuli. In Experiment 1, Group G.5, which did not receive discrimination training, failed to acquire as rapidly as Group GC, perhaps because incidental stimuli were not adequately neutralized.

Other sources of confounding are also evident. For example, Groups GC and GR differed in their discrimination performance during pretraining, indicating that, perhaps, the groups encountered discriminative $\mathrm{CS}-\mathrm{s}$ that were not equally discriminable from CS + . The effects of confounding discrimination difficulty or its behavioral by-products (e.g., between-groups differences in response rates to $\mathrm{CS}-$ ) upon subsequent performance in a retardation test are not clear. Interpretative difficulties introduced by the use of different pretreatments in the controlgroups design are successfully avoided by the use of a common pretraining regimen and a betweengroups generalization procedure that provides for group comparisons.

Experiment 2 used a discriminative autoshaping pretreatment identical to that administered to Group GC in Experiment 1. Subsequently, different groups of pigeons were administered reinforced autoshaping trials to different orientations of a white line. An incremental gradient of resistance to reinforcement with a trough at the value of the former CS - would provide evidence that the pretreatment generates inhibitory control by line orientation.

\footnotetext{
Method

Subjects. The subjects were 32 experimentally naive adult homing pigeons maintained at $75 \%$ of their free-feeding weights. Subjects were housed and maintained as in Experiment 1.

Apparatus. The apparatus was that utilized in Experiment 1. In addition, three filters were used in conjunction with miniature lamps to provide white lines $4 \mathrm{~mm}$ in diameter that bisected the response key at angles of $0 \mathrm{deg}$ (horizontal), $30 \mathrm{deg}$, and $60 \mathrm{deg}$.

Procedure. Subjects were unsystematically divided into four groups of eight pigeons each, and on Day 1 they were trained to approach and eat from the hopper. Pretraining for all subjects was identical to that for Group GC in Experiment 1. Test Day 1 began with a 10-trial warm-up, followed by 50 trials in which the CS was a white line that bisected an otherwise dark key. Each presentation of the white line was followed by $4 \mathrm{sec}$ of access to a tray of mixed pigeon grain. The orientation of the white line in the test phase differed for each of the four groups $(0,30,60$, and $90 \mathrm{deg}, 90 \mathrm{deg}$ being vertical). On Test Days 2-8, 60 reinforced presentations of the white line CS were administered.
}

\section{Results and Discussion}

During pretraining, there were no group differences in responding to either CS+ or CS- that approached significance. A criterion of responding on five consecutive trials was used to assess acquisition in the test phase. The mean and median number of trials to criterion for each group are plotted in Figure 2. As expected, the 0 -deg group exhibited the least resistance to reinforcement, followed by the 30 deg group. Acquisition to the 60-deg and 90-deg (former CS-) stimuli were comparable. A one-way analysis of variance on mean number of trials to criterion indicated a significant effect of groups $[F(3,28)=3.36, p<.05]$. That the gradient was orderly was indicated by a trend analysis on trials to criterion, which revealed a significant linear component to the gradient $[F(3,28)=8.32, p<.01]$, but a nonsignificant quadratic component $(\mathrm{F}<1)$.

The mean acquisition functions based on $\mathrm{CR}$ probability and CR frequency over blocks of 10 trials for each of the groups of subjects were consistent with the trials-to-criterion data. The 0-deg group reacquired the fastest, and the 60- and 90-deg groups were comparably retarded. Such acquisition differences were maintained only through the initial 140 trials, and postacquisition differences in asymptotic responding were not observed.

In summary, the data reveal that the retarding properties of the test stimuli are directly related to their similarity to the discriminative $\mathrm{CS}$ - along the dimension of orientation. The incremental gradient of resistance to reinforcement in the present experiment implies that the discriminative autoshaping procedure produces conditioned inhibition and, furthermore, that dimensional inhibitory control is exercised by the orientation of the CS-. These results reinforce the inhibitory interpretation of the retardation effect observed in Experiment 1, in that follow-

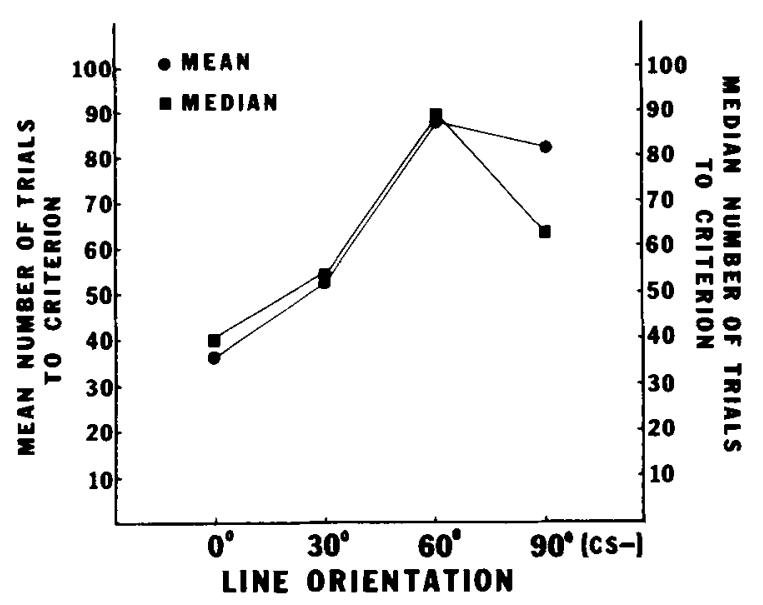

Figure 2. Mean and median number of trials to attain a criterion of responding on five consecutive presentations of the test $\mathrm{CS}$ for Groups 0, 30, 60, and 90 deg. 
ing a common history of pretraining, relative retardation of acquisition to the vertical line $\mathrm{CS}$ - was still observed.

\section{EXPERIMENT 3}

Experiment 2 demonstrated dimensional inhibitory control by orientation following discriminative autoshaping. The inhibitory generalization assessment procedures utilized in Experiment 2 differed from the usual generalization assessment methodology in two important ways. First, it has been common practice to obtain inhibitory gradients with extinction in effect at all values of the testing dimension, and second, generalization is usually assessed within subjects (i.e., each subject is tested for response strength at all values of the test dimension).

The resistance-to-extinction test of dimensional inhibitory control has been criticized for its failure to generate levels of responding that are adequate to allow assessment of the generalization tendency (Hearst, Besley, \& Farthing, 1970). Since the resistanceto-reinforcement procedure is not burdened by this consideration, it would appear to be a more reliable assessment vehicle. Unfortunately, the dimensional resistance-to-reinforcement test has been limited in its application to the free-operant domain (cf. Hearst et al., 1970; Karpicke \& Hearst, 1975; Rilling, Caplan, Howard, \& Brown, 1975; Besley \& Hearst, Note 2). The present experiments represent, to our knowledge, the first reports of the application of the methodology to Pavlovian procedures.

The technique generates an orderly between-subjects gradient (Experiment 2). The free-operant generalization studies that use the resistance-to-reinforcement test uniformly administer the test in a within-subjects design. The purpose of Experiment 3 was to determine the form of the within-subjects orientation gradient of resistance to reinforcement following discriminative autoshaping.

\footnotetext{
Method

Subjects. The subjects were 16 experimentally naive adult homing pigeons maintained at $75 \%$ of their free-feeding weights. Subjects were housed and maintained as in Experiment 1.

Apparatus. The apparatus consisted of four chambers similar to those utilized in Experiment 1. Masking noise ( $90 \mathrm{~dB}, \mathrm{SPL}$ ) was provided by the operation of ventilating fans.

Procedure. The subjects were unsystematically divided into two groups of eight pigeons each and were trained to approach and eat from the grain hopper. Subjects were given 14 daily sessions of discriminative autoshaping similar to the procedures employed during pretraining for Groups GC and GR in Experiment 1, with the following changes implemented. The CS duration was $7.5 \mathrm{sec}$. The US duration was $5.0 \mathrm{sec}$. Following discrimination pretraining, the two groups (GC and GR) entered the test phase of the experiment. Test Day 1 consisted of a 10-trial warm-up, followed by the administration of reinforced test trials in 4-trial blocks. Within each block, 0-, 30-, 60-, and 90-deg (vertical) white lines that bisected an otherwise dark response key were presented. Twelve
}

randomized blocks of four trials were presented on Test Day 1, and 15 randomized blocks were presented on Test Days 2 and 3.

\section{Results and Discussion}

There were no group differences in responding to either CS+ or CS- during the last 10 days of discriminative autoshaping, although, as in Experiment 1 , pigeons in Group GC responded more to CS- early in pretraining. Several different measures of test performance failed to show an orderly inhibitory gradient in Group GC. The mean percentage of total responses and mean absolute number of responses on Test Day 1 as a function of orientation are shown in Figure 3 (note the restricted range of the ordinates). The gradients are nonmonotonic and show modal responding to the 30-deg orientation. The absence of a systematic gradient was revealed by a repeatedmeasures analysis of variance with subjects and stimulus orientation as factors that failed to yield a reliable effect of stimulus orientation on either the absolute or the relative response measure (Fs $<1)$.

The conclusion that the gradient is not incremental is supported by two different criterion measures of acquisition. The first criterion measure is based on the number of trials before the pigeon pecked at a stimulus orientation in two consecutive blocks. A criterion score at each orientation was obtained from each pigeon; the mean gradients for Groups GC and GR are presented in Figure 4 (the GC-negAM group is discussed below). The figure reveals that Group GR initiated responding to all orientations sooner than did Group GC and that neither group achieved criterion acquisition to the four line orientations at differential rates (i.e., both gradients were flat). A twofactor mixed-design analysis of variance provided corroboration of this description, as the main effect of groups was significant $[F(1,14)=5.03, p<.05]$ but the effect of orientation and the Groups by

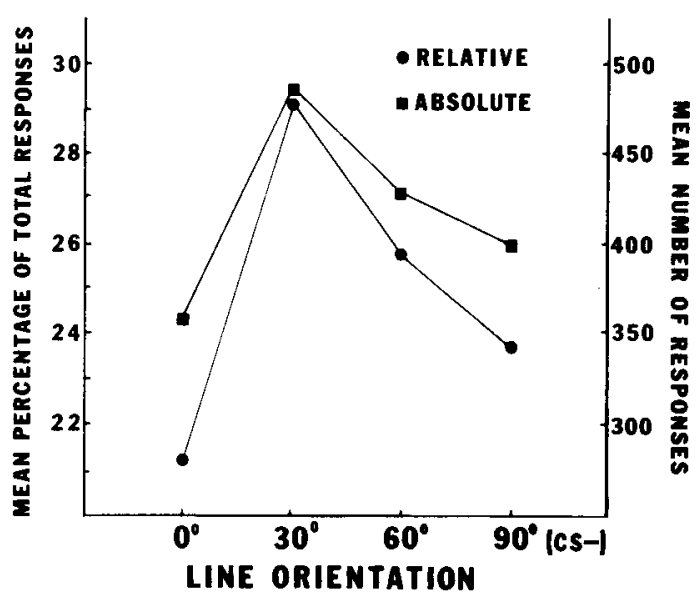

Figure 3. Mean percentage of total responses (relative gradient) and mean number of responses (absolute gradient) as a function of line orientation. 


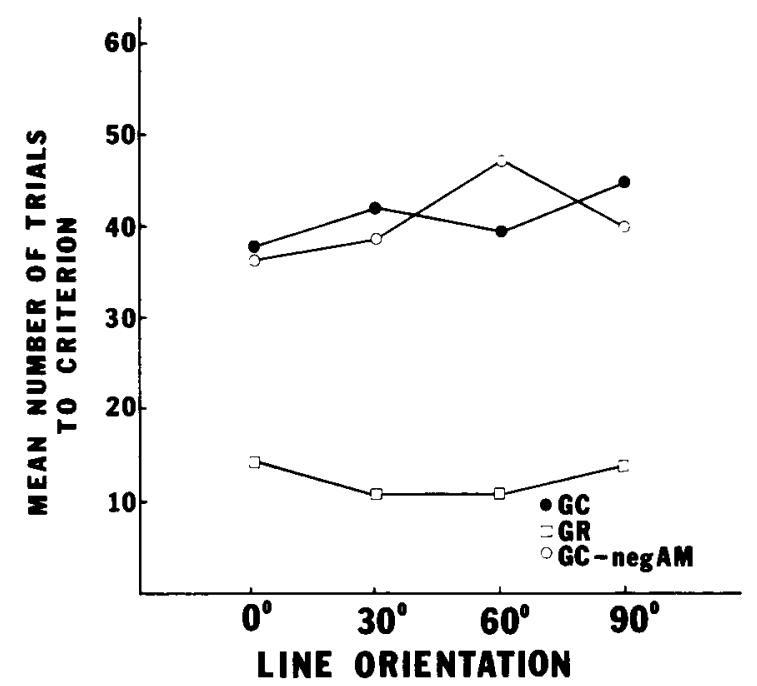

Figure 4. Mean number of test trials to a criterion of responding on two presentations of a particular stimulus orientation in two consecutive blocks as a function of stimulus orientation for Groups GC, GR, and GC-negAM.

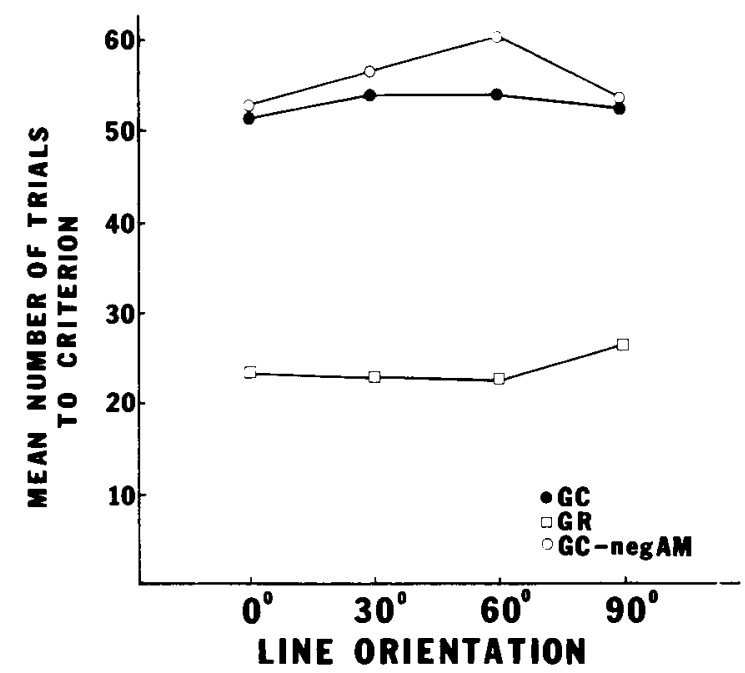

Figure 5. Mean number of test trials to a criterion of responding to a total of five presentations of a particular stimulus orientation as a function of line orientation for Groups GC, GR, and GC-negAM.

Orientation interaction were not $[\mathrm{F}(3,42)=2.50$, $p>.05$, and $F(3,42)=1.86, p>.05$, respectively]. Furthermore, an analysis of variance with subjects and orientation as factors on the criterion data of Group GC indicated that stimulus orientation had no significant effect $[F(3,21)=1.35, p>.05]$.

The second criterion measure evaluated was the number of trials to respond to a total of five presentations of a particular stimulus orientation. For each pigeon, a criterion score was obtained at each line angle. The mean criterion scores for each line angle for Groups GC and GR are presented in Figure 5. As the figure reveals, the retardation of the acquisi- tion of keypecking was not systematically affected by stimulus orientation. A repeated-measures analysis of variance confirmed the observation that acquisition to the various stimuli was not reliably affected by orientation in the GC group $(\mathrm{F}<1)$. Figure 5 also reveals that Group GR exhibited overall faster acquisition to all line orientations than did Group GC. A mixed-design analysis of variance with groups, subjects, and orientation as factors revealed a reliable main effect of groups $[F(1,14)=6.09, p<.05]$, but neither the effect of orientation nor the Groups by Orientation interaction was reliable $[F<1$ and $\mathrm{F}(3,42)=1.74, \mathrm{p}>.05$, respectively].

The data indicate that Group GR acquired keypecking to the test stimuli more rapidly than did Group GC; however, in neither group was acquisition differentially affected by line orientation. The group difference in acquisition is consistent with the results of Experiment 1 and suggests that the line component of the CS- is a conditioned inhibitor. The absence of an incremental orientation gradient in Group GC is inconsistent with the results of Experiment 1 and indicates that the orientation attribute of the line does not exercise dimensional inhibitory control.

The different measures consistently indicate that response acquisition was not influenced by the orientation of the test stimulus. The failure to obtain an inhibitory generalization gradient in the withinsubjects test is inconsistent with the results of Experiments 1 and 2 and contrary to the spirit of the freeoperant literature. Since the pretraining procedures for Group GC in Experiments 2 and 3 were almost identical (differing only in the source of masking noise and the duration parameters of CS and US), it seems reasonable to attribute the outcome discrepancy to the differences in the test procedure. In that regard, it should be noted that differential sensitivity of between- and within-subjects generalization procedures to excitatory dimensional control has been observed by Hiss and Thomas (1963) in the freeoperant realm. Hiss and Thomas reported that a within-subjects test for resistance to extinction in pigeons produced a flatter gradient than did the between-subjects procedure.

There are a number of factors inherent in the within-subjects testing procedure that might influence the slope of the gradient (see Mackintosh, 1974, pp. 494-498). Stimulus sequencing effects in a withinsubjects test allow for the occurrence of Pavlovian negative induction; that is, the tendency to respond on a given trial may be influenced by the nature of the immediately preceding trial. Such induction effects have been noted in free-operant investigations with pigeons by Donahoe, McCroskery, and Richardson (1970). In the present Experiment 3, the test stimuli were presented in blocks of trials such that the CS- 
was least likely to follow itself. Since CS - tends to potentiate responding on the subsequent trial, negative induction would tend to steepen rather than flatten the gradient. Nevertheless, the data were inspected for trial-by-trial sequencing effects, and no negative induction was discernible.

Another factor present in the within-subjects procedures used here is the asymmetry of the generalization test series. An asymmetrical generalization test series is known to generate a "central tendency effect" (cf. Giurantano, 1972; Thomas \& Jones, 1962; Thomas, Strub, \& Dickson, 1974). The term refers to the tendency for the gradient to peak at a point displaced from the training stimulus toward the center of the test series, resulting in the flattening of the gradient. The central tendency effect is generally observed to occur gradually over the course of testing; however, block-by-block inspection of the data revealed no evidence of the operation of such an influence. It is conceivable, on the other hand, that the effect precedes the initiation of responding, since exposure to several blocks of test stimuli generally occurs before autoshaping.

Inspection of the test data on a trial-by-trial basis revealed that the majority of the pigeons tended to respond to all stimuli once responding to any stimulus was initiated. Note that this all-or-none feature was also observed in subjects tested in the betweensubjects procedure, in which it was not at all troublesome. In the within-subjects procedure, however, such behavior obliterates the gradient. It is conceivable that the occurrence of the response per se affects subsequent responding, and this points to still another difference between the two generalization methodologies. The occurrence of the Pavlovian CR introduces CR-US contiguity, which would promote the intrusion of instrumental response acquisition. The development of instrumental keypecking would flatten only the within-subjects gradient.

\section{EXPERIMENT 4}

Temporal contiguity between the keypeck CR and the food US may promote instrumental keypecking, which would reduce the sensitivity of the withinsubjects generalization test. Contiguity between the $\mathrm{CR}$ and the US would be eliminated by implementing a differential reinforcement of other behavior (DRO) or negative automaintenance (cf. Williams \& Williams, 1969) contingency. The effects of a negative automaintenance contingency during testing on the slope of the orientation gradient of Group GC were investigated in Experiment 4.

\section{Method}

Subjects. The subjects were eight experimentally naive adult homing pigeons maintained at $75 \%$ of their free-feeding weights. Subjects were housed and maintained as in Experiment 1.
Apparatus. The apparatus was that used in Experiment 3.

Procedure. The subjects in this experiment were designated Group GC-negAM and were treated exactly as were pigeons in Group GC of Experiment 3, except that an omission contingency was in effect during testing. Responses during the test CS did not affect the CS but canceled the occurrence of the US.

\section{Results and Discussion}

Figure 4 presents criterion acquisition data for subjects in Group GC-negAM (criterion is attained upon responding to a stimulus on two consecutive blocks). Inspection of the figure suggests that retardation of acquisition of autoshaping was not systematically affected by stimulus orientation, an observation confirmed by the absence of an orientation effect in a repeated-measures analysis of variance $(\mathrm{F}<1)$. The gradients of Groups GC and GC-negAM did not reliably differ, as indicated by a mixed-design analysis of variance: Effects of groups (DRO contingency), orientation, and the Groups by Orientation interaction were not significant $(\mathrm{Fs}<1)$.

The second criterion measure (see Figure 5) yielded some evidence of differential acquisition to the test stimuli, and repeated-measures analysis of variance with subjects and orientation as factors revealed a reliable effect of stimulus orientation $[F(3,21)=3.52$, $\mathrm{p}<.05]$. A trend analysis on the GC-negAM gradient, however, revealed a nonsignificant linear component $(\mathrm{F}<1)$ and a significant quadratic component $[F(1,21)=8.39, p<.01]$. The significant nonlinear component to the gradient indicates that the gradient was not an orderly inhibitory one. A direct comparison of the gradients of Groups GC and GC-negAM revealed no reliable effect of groups $(F<1)$, orientation $[F(3,42)=2.80, p>.05]$, or the Groups by Orientation interaction $[F(3,42)=1.11, p>.05]$.

To evaluate the possibility that the initiation of responding is a function of orientation, an additional acquisition index based on the number of subjects that made the first test response to each orientation was examined. The 16 subjects in Groups GC and GC-negAM were combined, since the DRO contingency was not experienced until after the first response. The $0-, 30-, 60-$, and $90-\mathrm{deg}$ orientations were responded to first by five, four, three, and four subjects, respectively. A chi-square test revealed a nonsignificant effect of stimulus orientation $\left[\chi^{2}(3)=\right.$ $.125]$, once again indicating the absence of an inhibitory gradient.

\section{GENERAL DISCUSSION}

The four experiments reported here represent the application of different retardation or resistance-toreinforcement techniques to the study of inhibition in autoshaping. Experiments 1 and 2 utilized techniques based upon between-subjects comparisons, and they point to the development of inhibition to the distinctive 
line component of the discriminative CS- (Experiment 1), as well as dimensional inhibitory control by the orientation of the line element (Experiment 2). The results of Experiment 1 were replicated in Experiment 3; however, an incremental within-subjects orientation gradient was not discernible by any of a number of measures of $C R$ acquisition. The implementation of a DRO contingency during the withinsubjects generalization test (Experiment 4) failed to alter the form of the gradient.

In summary, discriminative autoshaping produces inhibition to the line component of the CS-. The between-subjects generalization gradient of orientation is incremental; however, the within-subjects gradient is not. The usefulness of the retardation metric in a within-subjects generalization test seems to be diminished by the tendency for subjects to respond to all stimuli once responding to any stimulus has been initiated. Since the addition of a DRO contingency during the test failed to resurrect the inhibitory gradient, it seems unreasonable to implicate the intrusiveness of instrumental acquisition factors as mediators of the discrepancy between test results.

The elevation and the flatness of the within-subjects gradient suggests that all of the orientations are inhibitory, and equally so. Explanations that might account for the flatness of the gradient (e.g., central tendency effect, disinhibition, instrumental acquisi(ion) are unable to adequately justify the elevation of the gradient, since they specify mechanisms for the breakdown rather than the maintenance of conditioned inhibition. The elevation and flatness of the within-subjects gradient also reiterate the distinction between inhibition and dimensional inhibitory control. A CS may be a conditioned inhibitor, but its attributes do not necessarily exercise dimensional stimulus control. Furthermore, the detection of dimensional inhibitory control may depend upon the assessment methodology employed.

\section{REFERENCE YOTES}

1. Lrcuioli, P. J. Discriminative autoshaping and inhibitory stimulus control. Paper presented at the meetings of the Eastern Psrichological Association. New York, 1976.

2. Besley. S., \& Hearst. E. Inhibitory gradients obtained with nondifferential reinforcement during generalization testing. Paper presented at the meeting of the Psychonomic Society. St. Louis, Missouri, 1969.

\section{REFERENCES}

Donahof, J. W., McCroskery, J. H., \& Richardson, W. K. Effects of context on the post discrimination gradient of stimulus generalization. Journal of Experimental Psychology, 1970. 84, 58-63.
Giurantano, L. P. Stimulus generalization in humans and pigeons as a function of training and test stimulus parameters. Unpubished doctoral dissertation, University of Colorado, 1972.

Hearst, E.. Besley, S., \& Farthing, G. W. Inhibition and the stimulus control of orerant behavior. Journal of the Experimental Analysis of Behavior, 1970, 14, 373-409.

Hearst, E., \& Franklin, S. R. Positive and negative relations between a signal and food: Approach-withdrawal behavior to the signal. Journal of Experimental Psychology: Animal Behavior Processes, 1977, 3, 37-52.

Hearst, E., \& Jenkins, H. M. Sign-tracking: The stimulusreinforcer relation and directed action. Austin. Tex: Psychonomic Society, 1974.

Hiss, R. H., \& Thomas, D. R. Stimulus generalization as a function of testing procedure and response measure. Journal of Experimental Psychology, 1963, 65, 587-592.

Honig, W. K. Attentional factors governing the slope of the generalization gradient. In R. M. Gilbert \& N. S. Sutherland (Eds.), Animal discrimination learning. London: Academic Press, 1969.

Jenkins, H. M., \& Harrison, R. H. Effect of discrimination training on auditory generalization. Journal of Experimental Psvchology, 1960, 59, 246-253.

Karpicke, J.. \& Hearst. E. Inhibitory control and errorless discrimination learning. Journal of the Experimental Analvsis of Behavior, 1975, 23, 159-166.

Mackintosh, N. H. The psychology of animal learning. New York: Academic Press. 1974.

Rescorla, R. A. Pavlovian conditioned inhibition. Psychological Bulletin, 1969, 72, 44-94.

Rilling, M., Caplan, H. J., Howard, R. C., \& Brown, C. H. Inhibitory stimulus control following errorless discrimination learning. Journal of the Experimental Analysis of Behavior, $1975,24,121-134$.

Thomas, D. R., \& Jones, C. G. Stimulus generalization as a function of the frame of reference. Journal of Experimental Psychology, 1962, 64, 77-80.

Thomas, D. R., Strub, H., \& Dickson, J. F. Adaptation-level and the central tendency effect in stimulus generalization. Journal of Experimental Psychology, 1974, 103, 466-474.

WaGiner, A. R. Incidental stimuli and discrimination learning. In R. M. Gilbert \& N. S. Sutherland (Eds.), Animal discrimina tion learning. London: Academic Press, 1969.

Wasserman, E. A., \& Anderson, P. A. Differential autoshaping to common and distinctive elements of positive and negative discriminative stimuli. Journal of the Experimental Analysis of Behavior, 1974. 22, 461-496.

Wasserman, E. A.. Franklin, S. R., \& Hearst, E. Pavlovian appetitive contingencies and approach versus withdrawal to conditioned stimuli in pigeons. Journal of Comparative and Phvsiological Psychology, 1974, 86, 616-627.

Wasserman, E. A., \& Molina, E. J. Explicitly unpaired key light and food presentations: Interference with subsequent autoshaped keypecking in pigeons. Journal of Experimental Psychology: Anmul Behavior Processes, 1975, 1, 30-38.

Wesseicls, M. G. Errorless discrimination, autoshaping, and conditioned inhibition. Science, 1973, 183, 941-943.

Williams. D. R., \& Williams, H. Automaintenance in the pigeon: Suctained pecking despite contingent nonreinforcement. Journal of the Experimental Analvsis of Behavior, 1969. 12. $511-520$

(Recels ed for publication July 12, 1979; (u) ion accepted April 29, 1980.) 